\title{
Kondo effect in a quantum dot coupled to ferromagnetic leads: A numerical renormalization group analysis
}

\author{
Mahn-Soo Choi, ${ }^{1}$ David Sánchez, ${ }^{2}$ and Rosa López ${ }^{2}$ \\ ${ }^{1}$ Department of Physics, Korea University, Seoul 136-701 Korea \\ ${ }^{2}$ Département de Physique Théorique, Université de Genève, CH-1211 Genève 4, Switzerland
}

(Dated: October 29, 2018)

\begin{abstract}
We investigate the effects of spin-polarized leads on the Kondo physics of a quantum dot using the numerical renormalization group method. Our study demonstrates in an unambiguous way that the Kondo effect is not necessarily suppressed by the lead polarization: While the Kondo effect is quenched for the asymmetric Anderson model, it survives even for finite polarizations in the regime where charge fluctuations are negligible. We propose the linear tunneling magnetoresistance as an experimental signature of these behaviors. We also report on the influence of spin-flip processes.
\end{abstract}

PACS numbers: 72.15.Qm, 72.25.Mk, 73.63.Kv

Introduction.-Magnetic impurities embedded in metallic hosts cause anomalous resonant scattering of conduction band electrons. At the same time, the localized magnetic moments are screened at low temperature by the itinerant electron spins. This is the celebrated Kondo effect [1], which has been recently revived in mesoscopic physics [2]. Ever since the theoretical predictions [3, 4] and the experimental demonstrations [5], the Kondo effect in phase-coherent systems such as quantum dots (QD's) has stimulated great interest in this field. The remarkable success behind this is the fine tunability of the parameter space (impurity level and hybridization couplings). The controlled manipulation in mesoscopic systems has not only allowed to test various aspects of the Kondo effect, which is a hard task in bulk solids, but also has posed further exciting questions. For example, when the spin-degeneracy of the impurity level is lifted by an external magnetic field, the Kondo peak in the density of states (DOS) of the dot is expected to split [6]. However, new experiments [7] and theoretical studies [8] suggest that the situation is more subtle.

A flood of very recent works $9,10,11,12,13,14$, 15, 16] has introduced another interesting issue, namely, how the Kondo physics is affected when the continuum electrons themselves are allowed to form spin-dependent bands. The motivation for this research stems from the successful field of spintronics [17]. In particular, a change has been detected in the resistivity of a Kondo alloy due to spin-polarized currents [18]. Furthermore, it is already possible to attach ferromagnetic leads to a carbon nanotube [19], and a carbon-nanotube QD has been shown to display Kondo physics below an unusually high temperature [20]. In addition, a QD coupled to ferromagnetic electrodes has been proposed as a promising candidate for spin injection devices, but so far, analyzed only in the Coulomb blockade regime 21]. The present work provides precise theoretical predictions in a wider region of the parameter space including the strong coupling limit.

The aim of our work is twofold. First, based upon a numerical renormalization group (NRG) calculation, we investigate the influence of ferromagnetic electrodes and the relative orientations of their magnetizations on the equilibrium properties of a QD with and without intrinsic spin flip processes. To the best of our knowledge, this is the first study of the model that sweeps across the different regimes (i.e., Kondo, mixed-valence, and emptyorbital), thoroughly analyzed with the assessment of local DOS, linear conductance, and tunneling magnetoresistance (TMR). Second, we resolve a controversy lately raised in the literature with regard to whether a spindependent renormalization of the impurity level induced by the spin-polarized leads will split the Kondo peak when the magnetic moments of both leads are aligned. In Ref. 9], an equation-of-motion (EOM) method plus an ansatz for the interacting self-energy [22] were employed and it was suggested that the splitting, $\delta$, is absent. In a later work 13], the scaling arguments (together with the EOM method) were used to find that $\delta$ is nonzero. In Ref. [10], using a similar approach, a splitting was predicted only in the mean-field peaks. In a more recent work [16], they made use of a noncrossing approximation $(\mathrm{NCA})$ and obtained $\delta \neq 0$. In Refs. 11, 12], on the other hand, the slave-boson mean-field theory (SBMFT) was utilized to study the zero-temperature properties and no splitting was observed. The answer to the controversy is, thus, elusive because each approximation method mentioned above has certain drawbacks of its own. Below, according to a NRG calculation, which has been known to provide very accurate results for impurity problems 23], we resolve clearly the controversy.

Our main result is summarized in Fig. 2(a). We find that in the presence of electron-hole symmetry the Kondo peak at the Fermi level remains unsplit even at finite polarizations and the linear conductance achieves the unitary limit. This remains true as long as only spin fluctuations are present in the QD. On the contrary, when charge fluctuations start to play a role (as in the asymmetric case of the Anderson model), the Kondo peak shows a visible splitting and the conductance is then suppressed.

Model.-We consider an ultrasmall tunnel junction 


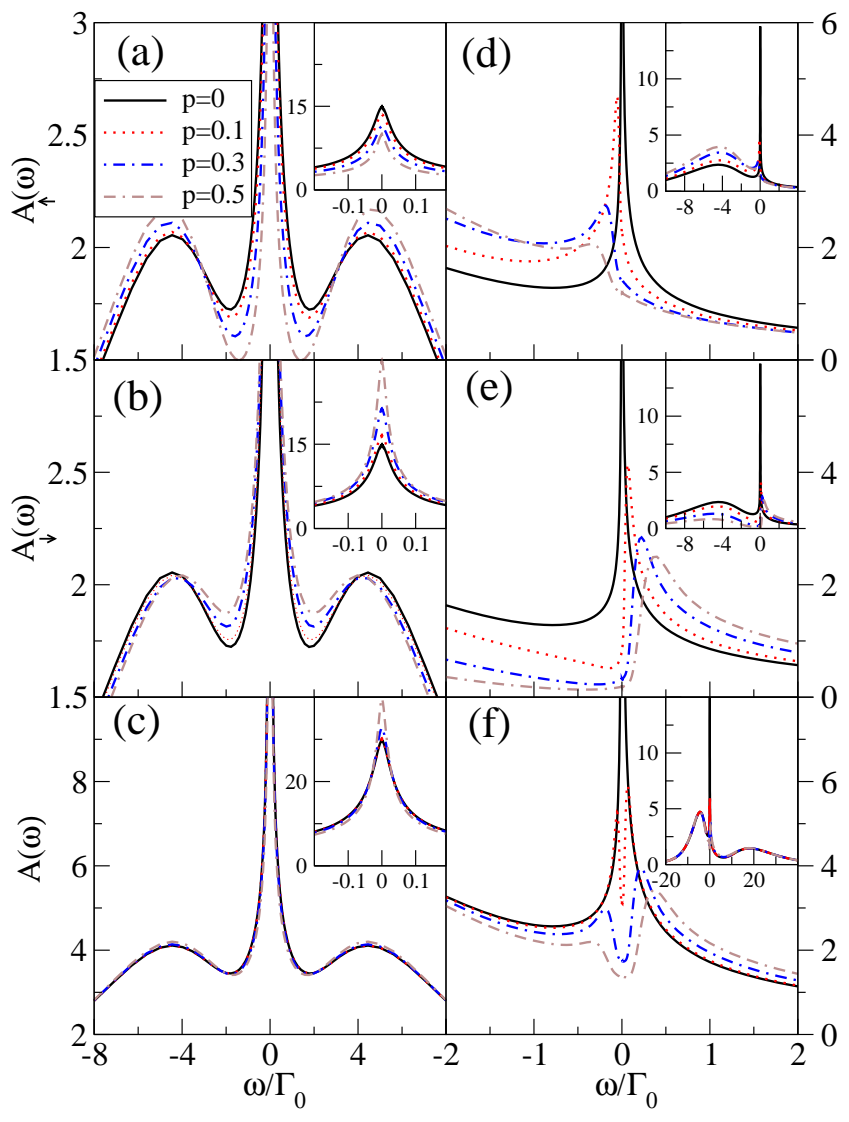

FIG. 1: Left: Local DOS of the QD for the symmetric Anderson model. (a) $A_{\uparrow}(\omega)$, (b) $A_{\downarrow}(\omega)$, and (c) $A(\omega)$ for $\varepsilon_{d}=-U / 2=-0.1 D$ ( $D$ is the bandwidth). Right: DOS for the asymmetric case. (d) $A_{\uparrow}(\omega),\left(\right.$ e) $A_{\downarrow}(\omega)$, and (f) $A(\omega)$ for $\varepsilon_{d}=-0.1 D$ and $U=0.45 D$. In all cases $\Gamma_{0}=0.02 D$.

comprising a QD coupled to two ferromagnetic leads. We assume that the QD consists of an energy level with an unpaired spin- $1 / 2$ electron and a charging energy $U$. This way, the QD is equivalent to an Andersontype impurity with single-particle energy $\varepsilon_{d, \sigma}$ for spin $\sigma=\{\uparrow, \downarrow\}[\underline{3}, \underline{4}]$. Notice that $\varepsilon_{d, \sigma}$ includes the Zeeman energy $\Delta_{Z} \equiv \varepsilon_{d, \uparrow}-\varepsilon_{d, \downarrow}$ of an external magnetic field. In what follows, we set $\Delta_{Z}=0$ in order to unmask possible spin-dependent renormalizations of the bare energy level purely due to coupling with the leads 24]. On the other hand, we shall include in a phenomenological way internal spin-flip scattering processes with rates $2 R / \hbar[25]$. Tunneling of electrons from the QD to the leads (reservoirs) $\alpha=\{L, R\}$ is described by the hopping integral $V_{\alpha k \sigma}$. The resulting Hamiltonian is given by:

$$
\begin{aligned}
\mathscr{H} & =\sum_{\sigma} \varepsilon_{d, \sigma} \hat{n}_{\sigma}+U \hat{n}_{\uparrow} \hat{n}_{\downarrow}+R\left(d_{\uparrow}^{\dagger} d_{\downarrow}+\text { H.c. }\right) \\
& +\sum_{\alpha k \sigma} \varepsilon_{\alpha k \sigma} c_{\alpha k \sigma}^{\dagger} c_{\alpha k \sigma}+\sum_{\alpha k \sigma}\left(V_{\alpha k \sigma} c_{\alpha k \sigma}^{\dagger} d_{\sigma}+\text { H.c. }\right)
\end{aligned}
$$

where $c_{\alpha k \sigma}^{\dagger}\left(c_{\alpha k \sigma}\right)$ is the creation (annihilation) operator for an electron with wave vector $k$ and spin in the electrode $\alpha$. The QD occupation number is $\hat{n}_{\sigma}=d_{\sigma}^{\dagger} d_{\sigma}\left[d_{\sigma}^{\dagger}\right.$ $\left(d_{\sigma}\right)$ creates (annihilates) an electron in the dot].

For definiteness, we shall take identical leads with chemical potentials $\mu_{L}=\mu_{R}=E_{F}$ and symmetric couplings (i.e., equal tunnel barriers). Ferromagnetism on the leads may be represented either by a spin-dependent DOS $\rho_{\alpha \sigma}(\omega)$ or by spin-dependent tunneling amplitudes $V_{\alpha k \sigma}$. We choose the latter for convenience but both pictures are formally equivalent provided we are interested just in the transport properties of the junction. In any case, the overall effect results in a spin-dependent hybridization parameter $\Gamma_{\alpha \sigma}(\omega) \equiv \pi \sum_{k}\left|V_{\alpha k \sigma}\right|^{2} \delta\left(\omega-\varepsilon_{\alpha k}\right)$. (We neglect proximity effects such as stray fields induced in the QD). A further simplification consists of neglecting the energy dependence of $\Gamma_{\alpha \sigma}(\omega)$, evaluating it at $\omega=E_{F}$ (wide band limit). In the following, we choose $E_{F}=0$ as the origin of energies. Therefore, one could define the spin polarization (close to the Fermi energy) at each lead as $p_{\alpha}=\left(\Gamma_{\alpha \uparrow}-\Gamma_{\alpha \downarrow}\right) /\left(\Gamma_{\alpha \uparrow}+\Gamma_{\alpha \downarrow}\right)$ with $-1 \leq p_{\alpha} \leq 1$. We consider parallel $(\mathrm{P})$ and antiparallel (AP) magnetizations of the two leads. In the $\mathrm{P}$ case $\left(p_{L}=p_{R} \equiv p\right)$, we have $\Gamma_{L \uparrow}=\Gamma_{R \uparrow}=(1+p) \Gamma_{0} / 2$ and $\Gamma_{L \downarrow}=\Gamma_{R \downarrow}=(1-p) \Gamma_{0} / 2$, where $\Gamma_{0} \equiv \Gamma_{\alpha \uparrow}+\Gamma_{\alpha \downarrow}$, whereas the AP case $\left(p_{L}=-p_{R} \equiv p\right)$ yields $\Gamma_{L \uparrow}=\Gamma_{R \downarrow}=$ $(1+p) \Gamma_{0} / 2$ and $\Gamma_{L \downarrow}=\Gamma_{R \uparrow}=(1-p) \Gamma_{0} / 2$.

In order to apply the NRG technique more efficiently, we map Eq. (11) onto an effective model with a single lead. This is achieved by means of a canonical transformation [3, 26]. In the $\mathrm{P}$ configuration it reads (we omit the index $k$ for simplifying notation):

$$
c_{L \sigma}=\left(a_{\sigma}+b_{\sigma}\right) / \sqrt{2}, \quad c_{R \sigma}=\left(a_{\sigma}-b_{\sigma}\right) / \sqrt{2} .
$$

For AP polarizations one uses the relations:

$$
\begin{array}{ll}
c_{L \uparrow}=\left(V_{a} a_{\uparrow}+V_{i} b_{\uparrow}\right) / \mathcal{V}, & c_{R \uparrow}=\left(V_{i} a_{\uparrow}-V_{a} b_{\downarrow}\right) / \mathcal{V}, \\
c_{L \downarrow}=\left(V_{i} a_{\downarrow}+V_{a} b_{\downarrow}\right) / \mathcal{V}, & c_{R \downarrow}=\left(V_{a} a_{\downarrow}-V_{i} b_{\downarrow}\right) / \mathcal{V},
\end{array}
$$

where $V_{a}=V_{L \uparrow}=V_{R \downarrow}\left(V_{i}=V_{L \downarrow}=V_{R \uparrow}\right)$ is the tunneling amplitude for majority (minority) spins and $\mathcal{V}=$ $\sqrt{\left|V_{a}\right|^{2}+\left|V_{i}\right|^{2}}$. Substituting Eqs. (2)-(3) into Eq. (1) one can show that (i) the QD electron decouples from the $b_{\sigma}$ operators and hybridizes only with the quasiparticles described by the $a_{\sigma}$ operators; and (ii) the effective dot-lead couplings are renormalized for both configurations.

We use the NRG method to obtain the local DOS $A_{\sigma}(\omega)=A_{\sigma}^{>}(\omega)-A_{\sigma}^{<}(\omega)$ on the QD (i.e., at the impurity site). The particle and hole branches, $A_{\sigma}^{>}(\omega)$ and $A_{\sigma}^{<}(\omega)$, are defined by

$$
A_{\sigma}^{>/<}(\omega)= \pm \sum_{m}\left|\left\langle m\left|d_{\sigma}^{\dagger} / d_{\sigma}\right| 0\right\rangle\right|^{2} \delta\left(\omega \mp E_{m} \pm E_{0}\right),
$$

where $|0\rangle$ is the many-body ground state (electrodes + $\mathrm{QD})$ and $|m\rangle$ is an excited state ( $E_{0}$ and $E_{m}$ are the corresponding energies). Notice that all the physics (correlations, dependence on the gate voltage, etc.) is contained 

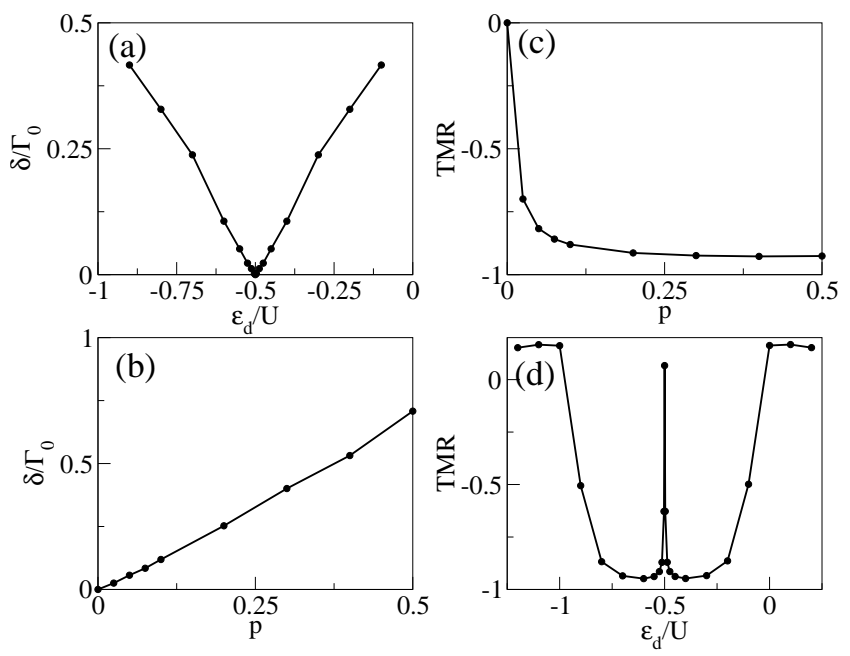

FIG. 2: (a) Splitting $\delta$ of the Kondo peak as a function of $\varepsilon_{d}$ for $p=0.25$ and $U=0.4 D$. (b) $\delta$ versus $p$ for $\varepsilon_{d}=-0.1 D$ and $U=0.45 D$. (c) TMR versus $p$ for $\varepsilon_{d}=-0.1 D$ and $U=0.4 D$. (d) TMR as a function of $\varepsilon_{d}$ for $p=0.25$ and $U=0.4 D$. In all cases $\Gamma_{0}=0.02 D$.

in $A_{\sigma}(\omega)$. The linear conductance (normalized to $e^{2} / h$ ) of the junction at zero temperature is obtained from the impurity spectral density function at the Fermi level 27], $g=\sum_{\sigma} 2 \Gamma_{L \sigma} \Gamma_{R \sigma} A_{\sigma}(0) /\left(\Gamma_{L \sigma}+\Gamma_{R \sigma}\right)$. As it is the case in usual Kondo experiments on QD's, we shall present results for large interaction $(U \gg \Gamma)$.

Results. - We first address the issue whether the Kondo peak in local DOS splits or not. (Until later, we put $R=0$.) Figure 11 shows $A_{\uparrow}(\omega), A_{\downarrow}(\omega)$, and $A(\omega)=$ $A_{\uparrow}(\omega)+A_{\downarrow}(\omega)$ for different values of the lead polarization $p$ in the $\mathrm{P}$ configuration. [The AP case is less interesting as both spin orientations are equally coupled after the transformation given by Eq. (3)]. Left panels correspond to the symmetric Anderson model (i.e., $\varepsilon_{d}=-U / 2$ ). For $p=0$, in addition to two (symmetric) mean-field peaks at $\omega=\varepsilon_{d}$ and $\omega=\varepsilon_{d}+U, A(\omega)$ shows a peak at $\omega=$ 0 [see Fig. 1(c)], which is responsible for the observed zero-bias anomaly. As $p$ increases, the spectral peak of $A_{\uparrow}(\omega)\left[A_{\downarrow}(\omega)\right]$ at the Fermi energy increases (decreases) [see insets of Fig. 11 (b) and (c)]. Remarkably, however, the central peaks of both $A_{\uparrow}(\omega)$ and $A_{\downarrow}(\omega)$ are pinned at the Fermi level; in particular, the Kondo peak in $A(\omega)$ does not split. Experimentally, one would see a perfect transparency of the junction (see below). Right panels of Fig. 1 show the same functions for the asymmetric case $\left(\varepsilon_{d} \neq-U / 2\right)$, where charge fluctuations are allowed to certain extent. As $p$ increases, $A_{\uparrow}$ and $A_{\downarrow}$ shift in opposite directions [see Fig. 1(d) and (e)] and the Kondo peak in $A(\omega)$ splits into two [Fig. 1(f)]. As a result, the Kondo effect is suppressed. We have checked as well that both mean-field peaks are shifted in opposite directions, though their strengths differ and the splitting cannot be resolved in Fig. 团f).
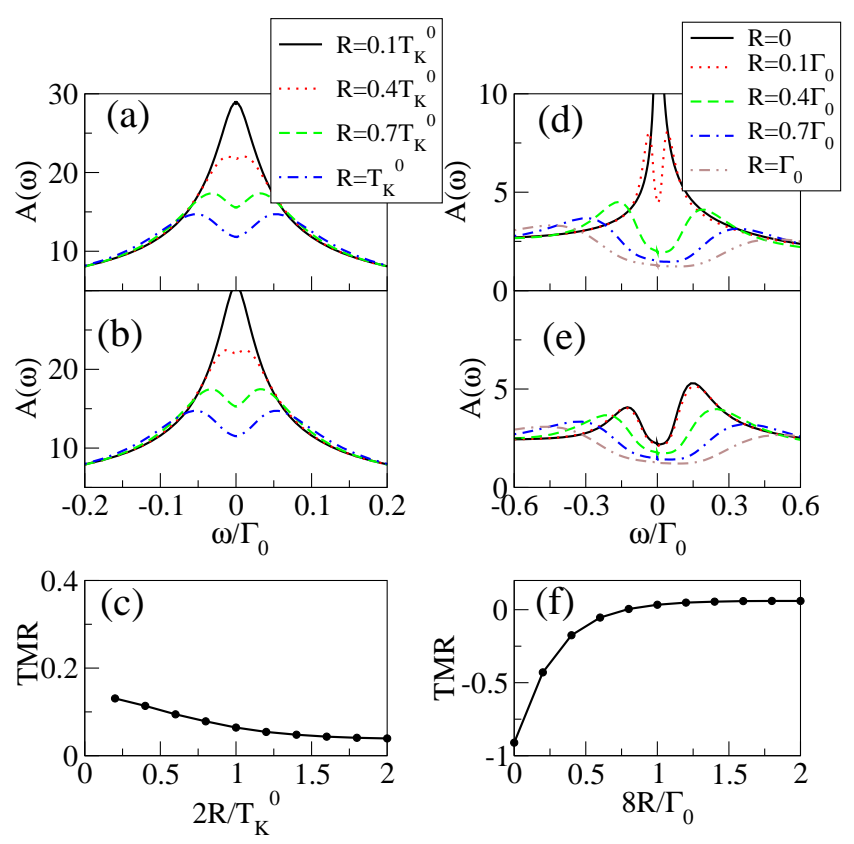

FIG. 3: The effects of spin-flip processes $R$ for the symmetric (left panels with $\varepsilon_{d}=-U / 2=-0.1 D$ ) and asymmetric (right panels with $\varepsilon_{d}=-0.1 D$ and $U=0.4 D$ ) Anderson model. Local DOS for (a,d) $p=0$ and (b,e) $p=0.25$. (c,f) TMR versus $R$ for $p=0.25$. In all cases $\Gamma_{0}=0.02 D$.

The finite splitting for the asymmetric Anderson model may be understood in terms of simple scaling arguments [13]: Because the hybridization for up spins is larger than for down spins $\left(\Gamma_{\alpha \uparrow}>\Gamma_{\alpha \downarrow}\right)$, the renormalization of the bare level $\varepsilon_{d}$ is spin-dependent; the $\uparrow(\downarrow)$ electron lowers (raises) its energy. Then, the coupling acts as an effective magnetic field, leading to a finite $\delta$ [28]. Yet, the perturbative nature of a poor man's scaling cannot describe the fixed point in the strong coupling regime. In particular, such simple scaling arguments cannot account properly for the particle-hole symmetry in the symmetric Anderson model and always predict $\delta \neq 0$. For the symmetric Anderson model, it is important to notice that the particle-hole symmetry quenches charge fluctuations completely for both spins $\left(\left\langle n_{\uparrow}\right\rangle=\left\langle n_{\downarrow}\right\rangle=1 / 2\right)$ at any $|p|<1$, and the real part of the self-energy (at $E_{F}$ ) is zero. This means that although the binding energy of the singlet state (the Kondo temperature $T_{K}$ ) diminishes with $p$, the quasiparticle lifetime is still infinite and the Fermi liquid picture is valid. Therefore, the results in Fig. 1(c) are consistent with SBMFT, which describes the Kondo peak when spin fluc-

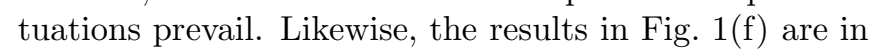
agreement with EOM and NCA models, which support charge fluctuations to some degree. Of course, the NRG method can encompass the whole parameter range.

To illustrate our conclusions, we measure the splitting $\delta$ of the Kondo peak as a function of $\varepsilon_{d}$ (experimentally this is controlled by a gate voltage) with $U$ fixed 
[see Fig. 2(a)]. The splitting $\delta$ increases roughly linearly from zero as moving away from the symmetric point $\varepsilon_{d}=-U / 2$. Notice also that well away from $\varepsilon_{d}=-U / 2$, $\delta$ is linear in the lead polarization [see Fig. 2(b)], confirming the prediction relying upon scaling arguments.

We now turn to the tunneling magnetoresistance of the system, defined by TMR $=\left(g^{P}-g^{A P}\right) / g^{A P}$. One can show that $g^{P}=\pi \Gamma_{0}\left[(1+p) A_{\uparrow}(0)+(1-p) A_{\downarrow}(0)\right]$ and $g^{A P}=\left(1-p^{2}\right) \pi \Gamma_{0} A(0)$ are the dimensionless linear conductances for the $\mathrm{P}$ and $\mathrm{AP}$ configurations, respectively. For the symmetric Anderson model, the Kondo effect survives even for a finite value of polarization $p(|p|<1)$, and $g^{P}$ preserves the unitary limit. As a result, the TMR is given by TMR $=p^{2} /\left(1-p^{2}\right)$. For the asymmetric Anderson model, on the contrary, $g^{P}$ gets strongly suppressed as $p$ increases. Then, the system exhibits a strong negative TMR [see Fig. 2(c)] 29]. Figure 2(d) shows TMR as a function of $\varepsilon_{d}$, which shows a sharp peak around the symmetry point $\left(\varepsilon_{d} \simeq-U / 2\right)$. The width of the peak is determined by how fast the Kondo effect is suppressed as $\left|\varepsilon_{d}-U / 2\right|$ increases from zero, and hence depends strongly on the polarization $p$ and the hybridization $\Gamma_{0}$; see Figs. 2] (a) and (b). Experimentally, finite temperatures would smoothen this peak.

So far we ignored the spin-flip scattering. $R \neq 0$ introduces a new degree of freedom in the QD, explicitly lifting the level degeneracy $\left(\varepsilon_{d} \pm R\right)$. In Fig. B(a,b) we plot the local DOS in the symmetric case for different values of $R$ in terms of the Kondo temperature $k_{B} T_{K}^{0}=\sqrt{\Gamma_{0} U / 2} \exp \left[-\pi\left|\varepsilon_{d}\left(\varepsilon_{d}+U\right)\right| / 2 \Gamma_{0} U\right]$. For $p=0$ the Kondo peak shrinks [30] and splits as expected (the effect can be ascribed to an external Zeeman field). For $p=0.25$ the splitting takes place for a smaller value of $R / T_{K}^{0}$. As a result, $T_{K}$ in the $\mathrm{P}$ case is always lesser than $T_{K}^{0}$ and TMR decreases in magnitude with increasing $R$ [see Fig. 3(c)]. The DOS for the asymmetric Anderson model are shown in Fig. 3(d,e) as a function of $R / \Gamma_{0}$ as here we are interested in the competition between both splitting factors $(p$ and $R$ ). For a nonzero value of $p$, the splitting is not symmetric unlike Fig. 3(b). This is again a consequence of the presence of charge fluctuations. For $R=0$ the DOS in the $\mathrm{P}$ alignment is already quenched due to the spin dependent coupling, which leads to a strong negative TMR. However, this effect is washed out with increasing $R$ and TMR tends to vanish [see Fig. [3(f)].

Conclusion.-Using a NRG method, we have shown that the Kondo effect in a quantum dot is not necessarily suppressed by the spin polarizations of the leads: for the symmetric Anderson model, where charge fluctuations are completely suppressed, the Kondo effect is robust even for finite polarizations. For the asymmetric Anderson model, the Kondo peak does split into two. We also reported on the TMR in the Kondo, mixed-valence, and empty-level regimes, being strongly affected in the presence of spin flip processes. The physics addressed in this paper is realistic and can be, in principle, tested with present techniques.

We thank M. Büttiker, T. Costi and K. Kang for valuable comments. M.-S.C. acknowledges supports from the SKORE-A program, from the eSSC at POSTECH, and from a Korea University Grant. D.S. and R.L. were supported by the Swiss NSF through the program MANEP, and by the Spanish MECD.

Note Added.-In the final stages of this work we became aware of a closely related work by J. Martinek et al., cond-mat/0304385. The difference is that they study only the asymmetric Anderson model, and focus rather on the restoring effect of an external magnetic field.

[1] A.C. Hewson, The Kondo Problem to Heavy Fermions (Cambridge University Press, Cambridge, 1993).

[2] L. Kouwenhoven and L. Glazman, Phys. World 14, 33 (2001).

[3] L.I. Glazman and M.E. Ră̈kh, Pis'ma Zh. Eksp. Teor. Fiz. 47, 378 (1988), [JETP Lett. 47, 452 (1988)].

[4] T.K. Ng and P.A. Lee, Phys. Rev. Lett. 61, 1768 (1988).

[5] D. Goldhaber-Gordon et al., Nature (London) 391, 156 (1998); S.M. Cronenwett, et al., Science 281, 540 (1998); J. Schmid et al., Physica B 256-258, 182 (1998).

[6] Y. Meir and N. S. Wingreen, Phys. Rev. Lett. 70, 2601 (1993); T. Costi, Phys. Rev. Lett. 85, 1504 (2000).

[7] S. Sasaki et al., Nature (London) 405, 764 (2000).

[8] M. Pustilnik et al., Phys. Rev. Lett. 84, 1756 (2000); M. Eto and Y.V. Nazarov, ibid. 85, 1306 (2000).

[9] N. Sergueev et al., Phys. Rev. B 65, 165303 (2002).

[10] P. Zhang et al., Phys. Rev. Lett. 89, 286803 (2002).

[11] B.R. Bułka and S. Lipiński, Phys. Rev. B 67, 024404 (2003).

[12] R. López and D. Sánchez, Phys. Rev. Lett. 90, 116602 (2003).

[13] J. Martinek et al., cond-mat/0210006 (unpublished).

[14] R. Lü and Z-R. Liu, cond-mat/0210350 (unpublished).

[15] J. Ma et al., cond-mat/0212645 (unpublished).

[16] B. Dong et al., cond-mat/0302372 (unpublished).

[17] See, e.g., S.A. Wolf et al., Science 294, 1488 (2001).

[18] T. Taniyama et al., Phys. Rev. Lett. 90, 016601 (2003).

[19] K. Tsukagoshi et al., Nature (London) 401, 572 (1999).

[20] J. Nygård et al., Nature (London) 408, 342 (2000).

[21] M.M. Deshmukh and D.C. Ralph, Phys. Rev. Lett. 89, 266803 (2002)

[22] T.K. Ng, Phys. Rev. Lett. 70, 3635 (1993).

[23] K.G. Wilson, Rev. Mod. Phys. 47, 773 (1975). H.R. Krishna-murthy et al., Phys. Rev. B 21, 1003 (1980); T.A. Costi et al., J. Phys.: Condens. Matter 6, 2519 (1994). W. Hofstetter, Phys. Rev. Lett. 85, 1508 (2000).

[24] The contribution from finite $\Delta_{Z}$ to the spin-dependent Kondo peak is (almost) independent of $\delta$ and can be used to compensate the latter; see Refs. [13] and [28].

[25] Note that this term does not involve a real energy relaxation mechanism. In an actual experiment, it could be accounted for by a transverse magnetic field or by a tunable spin-orbit coupling of the Rashba-Dresselhaus type.

[26] M. Büttiker, IBM J. Res. Developm. 32, 63 (1988). 
[27] Y. Meir and N.S. Wingreen, Phys. Rev. Lett. 68, 2512 (1992).

[28] M.-S. Choi, N. Y. Hwang, and S.-R. E. Yang, Phys. Rev. B 67, 245323 (2003).

[29] This should not be confused with the behavior of tunnel junctions with a noninteracting resonant level, where one usually has $g^{P}>g^{A P}$ and TMR increases with $p$ [see, e.g., A.M. Bratkovsky, Phys. Rev. B 56, 2344 (1997)].

[30] Had we dealt with inelastic spin-flip scattering, this reduction would have been stronger. A. Kaminski et al., Phys. Rev. Lett. 83, 384 (1999); R. López et al., Phys. Rev. B 64, 075319 (2001). 